\title{
The running coupling of QCD with four flavors
}

\author{
ALPHA Collaboration \\ Fatih Tekin ${ }^{\text {a }}$, Rainer Sommer ${ }^{\mathrm{b}}$, Ulli Wolff ${ }^{\mathrm{a}, *}$ \\ a Institut für Physik, Humboldt Universität, Newtonstr. 15, 12489 Berlin, Germany \\ b NIC, DESY, Platanenallee 6, 15738 Zeuthen, Germany
}

Received 4 June 2010; accepted 5 July 2010

Available online 11 July 2010

\begin{abstract}
We have calculated the step scaling function and the running coupling of QCD in the Schrödinger functional scheme with four flavors of $\mathcal{O}(a)$ improved Wilson quarks. Comparisons of our non-perturbative results with 2-loop and 3-loop perturbation theory as well as with non-perturbative data for only two flavors are made.
\end{abstract}

(c) 2010 Elsevier B.V. All rights reserved.

\section{Introduction}

One ingredient of the contemporary Standard Model is QCD which is believed to describe the strong interaction between quarks and gluons. The theory has six free mass parameters for the six quark species and one coupling parameter. After fixing these free parameters of the QCD Lagrangian at some reference energy scale, the theory in principle predicts presently known effects in which only the strong interaction is involved. Since QCD possesses the property of asymptotic freedom, perturbation theory is applicable in the high energy regime $(E \gg 1 \mathrm{GeV})$, but for low energies lattice QCD, the only known systematic non-perturbative approach, is required. Here a certain number of physical quantities, for example meson masses, have to be identified with experimental inputs to determine the free parameters and in particular to gauge dimensionful quantities on the lattice in powers of $\mathrm{GeV}$. Only then predictions become possible. As an exam-

\footnotetext{
* Corresponding author.

E-mail addresses: fatih@ physik.hu-berlin.de (F. Tekin), rainer.sommer@ desy.de (R. Sommer), uwolff@physik.hu-berlin.de (U. Wolff).
} 
ple, the light hadron mass spectrum was studied by different collaborations in quenched as well as in full QCD with up to three dynamical fermion species [1-6]. The theoretical predictions for the spectrum in full QCD are compatible with the experiments at the error-level achieved so far.

At a first glance the perturbative and non-perturbative formulations seem to be disjoint and have their own domains of applicability. In a long-term project, the ALPHA Collaboration has been developing methods and tools to connect these regimes by computing the parameters of the perturbative domain starting from the non-perturbative formulation [7]. In this context, the step scaling function which we will discuss later plays a key role. For different numbers of flavors, the step scaling function [8-13] which can be understood as an integrated form of the QCD $\beta$-function and the running coupling have been examined and determined successfully (see [1416] for recent publications). In this paper, we want to calculate the step scaling function and the running coupling with four flavors of $\mathcal{O}(a)$ improved Wilson quarks. For the present work, the improvement coefficient $c_{\mathrm{sw}}$ which is essential for the $\mathcal{O}(a)$ improvement with Wilson quarks was determined in a preceding paper [17]. We exploit this result in the simulations that we report in the following.

The paper is organized as follows. In Sections 2-5 we summarize some background about the non-perturbative definition of a coupling constant in the Schrödinger functional finite volume renormalization scheme and about the step scaling technique. In Section 6 we present our new raw data and their analysis. We will arrive, for four flavors, at a value for the dimensionless combination $\Lambda L_{\max }$. While $\Lambda$ parameterizes the coupling at high energy, $L_{\max }$ is an unambiguously defined length in the hadronic regime. Its value in $\mathrm{GeV}$ remains to be determined by large volume simulations.

\section{The finite size strategy}

The fundamental parameters of QCD, i.e. the coupling and masses of the quarks, depend on a scale $\mu$. To make contact with perturbative QCD we need to know their values for large $\mu$ in the domain of asymptotic freedom. If such a computation is attempted on a single lattice where at the same time hadronic scales are measurable with small finite volume and discretization effects one has to satisfy to a reasonable precision the string of inequalities

$$
L \gg \frac{1}{m_{\pi}} \approx \frac{1}{0.14 \mathrm{GeV}} \gg \frac{1}{\mu} \approx \frac{1}{10 \mathrm{GeV}} \gg a .
$$

In total this implies $L / a \gg 70$ which is impossible to satisfy for some time. A way to circumvent these difficulties was hence proposed by Lüscher et al. in [18]. The idea is to exploit the universality of the finite volume continuum limit to perform intermediate renormalizations with the finite size as a renormalization scale, $\mu=1 / L$. In these steps only $L \gg a$ has to be assured to render cutoff effects small. By such simulations one can non-perturbatively determine the change of the coupling constant in the continuum limit under scale changes from $L$ to $L / 2$. By repeating such steps we start at $L_{\max } \approx \mathcal{O}(0.5 \mathrm{fm})$ and arrive after $k$ steps at $\mu=2^{k} / L_{\max }$. With a sufficient number of steps this will be in the perturbative regime where we can make contact with the $\Lambda$ parameter of an arbitrary scheme and with the scheme independent renormalization group invariant quark masses by applying perturbative formulas. If one finally succeeds in computing the precise value of $L_{\max }$ in units of a mass or decay constant, then all references to the intermediate finite volumes will have disappeared from the final result. More details may be found in [7]. 


\section{Running coupling}

As mentioned before, the coupling and the quark masses are renormalization scale dependent and run with energy. Therefore the quoted values of the quark masses and the world average of $\alpha_{\mathrm{s}}$, both in the $\overline{\mathrm{MS}}$ scheme, in the Particle Physics Booklet [19] are referred to a particular reference scale ( $\mu \approx 2 \mathrm{GeV}$ for the masses and $\mu=M_{\mathrm{Z}}$ for the coupling).

From the theoretical point of view, the running of the QCD parameters is described by the renormalization group equation. However, a physical observable $\mathscr{O}$ should have no reference to a particular renormalization scale $\mu$. This fact is expressed by the Callan-Symanzik equation

$$
\left[\mu \frac{\partial}{\partial \mu}+\beta(\bar{g}) \frac{\partial}{\partial \bar{g}}+\tau(\bar{g}) \sum_{i=1}^{N_{\mathrm{f}}} \bar{m}_{i} \frac{\partial}{\partial \bar{m}_{i}}\right] \mathscr{O}=0 .
$$

In words: for any change of $\mu$ there are accompanying modifications of $\bar{g}$ and $\bar{m}_{i}$ such that the physics is unchanged. The implied scale dependence of the coupling is given by $\beta(\bar{g})$ in (3.1)

$$
\beta(\bar{g})=\mu \frac{\partial \bar{g}(\mu)}{\partial \mu} .
$$

For high energies or for weak couplings, the $\beta$-function has the following asymptotic expansion

$$
\beta(\bar{g}) \stackrel{\bar{g}}{=}=0
$$

The first two (1- and 2-loop) coefficients $b_{0}$ and $b_{1}$ in (3.3) are scheme independent

$$
\begin{aligned}
& b_{0}=\frac{1}{(4 \pi)^{2}}\left(11-\frac{2}{3} N_{\mathrm{f}}\right), \\
& b_{1}=\frac{1}{(4 \pi)^{4}}\left(102-\frac{38}{3} N_{\mathrm{f}}\right) .
\end{aligned}
$$

The 3-loop coefficient does depend on the scheme and in the Schrödinger functional scheme, which we will need later, $b_{2}$ has been given in [24]

$$
b_{2}=\frac{1}{(4 \pi)^{3}}\left[0.483(7)-0.275(5) N_{\mathrm{f}}+0.0361(5) N_{\mathrm{f}}^{2}-0.00175(1) N_{\mathrm{f}}^{3}\right] .
$$

We restrict ourselves to mass-independent schemes where all renormalization conditions are imposed at vanishing quark masses. Examples are the $\overline{\mathrm{MS}}$ scheme of dimensional regularization and the Schrödinger functional scheme used here. For $N_{\mathrm{f}} \leqslant 16$, the $\beta$-function (3.3) is negative at weak coupling and the integration of (3.2) results in a coupling which decreases with increasing energy. In other words, the quarks behave like free particles in the high energy regime (asymptotic freedom). But for $N_{\mathrm{f}}>16$, there is a sign change and the property of asymptotic freedom is lost. The relation of $\bar{g}(\mu)$ to the $\Lambda$ parameter of QCD is given by the following solution of the CallanSymanzik equation

$$
\Lambda=\mu\left[b_{0} \bar{g}^{2}(\mu)\right]^{-\frac{b_{1}}{2 b_{0}^{2}}} \exp \left\{-\frac{1}{2 b_{0} \bar{g}^{2}(\mu)}\right\} \exp \left\{-\int_{0}^{\bar{g}(\mu)} d x\left[\frac{1}{\beta(x)}+\frac{1}{b_{0} x^{3}}-\frac{b_{1}}{b_{0}^{2} x}\right]\right\} .
$$

As is well-known, $\Lambda$ is scheme-dependent but the transformation to other schemes follows from the relation between their couplings at one loop accuracy. 


\section{Coupling in the SF scheme}

The finite-volume scheme which we use for our simulations is the Schrödinger functional (SF) scheme. In the following, we will only give a brief reminder of the main features and properties of this scheme which are discussed in detail in many papers, for example [20-24].

The original proposal was made in [20]. The construction was guided by several design criteria. The coupling constant was to be defined independently of perturbation theory, but its evaluation had to be manageable both in lattice perturbation theory (also beyond one loop) and by numerical simulation. Moreover, small lattice artefacts were demanded. These requirements are not easy to fulfill and have led to the Schrödinger functional which is the Euclidean propagation kernel of a field configuration $C$ at time $x_{0}=0$ to another field configuration $C^{\prime}$ at time $x_{0}=T$. The free energy or effective action $\Gamma$ of such a system is given by

$$
Z\left[C^{\prime}, C\right]=\exp \{-\Gamma\}=\int_{\mathrm{V}} \mathscr{D}[U, \bar{\Psi}, \Psi] \exp \{-S[U, \bar{\Psi}, \Psi]\} .
$$

The action $S$ is defined as in [17]. The choice of $C_{k}$ and $C_{k}^{\prime}$ of the boundary gauge fields

$$
\begin{aligned}
& \left.U(x, k)\right|_{x_{0}=0}=\exp \left\{a C_{k}\right\}, \\
& \left.U(x, k)\right|_{x_{0}=T}=\exp \left\{a C_{k}^{\prime}\right\}
\end{aligned}
$$

is largely arbitrarily. After some optimization with regard to lattice artefacts constant Abelian fields turned out to be appropriate. We will also adopt this choice of boundary fields parameterized by the scale $L$ and two dimensionless real parameters $\eta$ and $v$ [8]. Numerical simulations of the Schrödinger functional showed that the choice $v=0$ leads to small statistical errors for the coupling in simulations. Therefore, we will also set $v$ to zero.

The Schrödinger functional coupling is now defined by the response of $\Gamma$ to a variation of the boundary fields around point 'A' of [8] via the parameter $\eta$,

$$
\Gamma^{\prime}=\left.\frac{\partial \Gamma}{\partial \eta}\right|_{\eta=0}=\frac{k}{\bar{g}^{2}}
$$

where $k$ is a normalization constant chosen such that the perturbative expansion of $\Gamma^{\prime}$ begins with the bare coupling at tree level [8]. It should be noticed that the only external scale which appears in the definition of the coupling is the box size $L$, i.e. recursive finite size techniques can be used for the investigation of the evolution of the coupling.

The key quantity $\Gamma^{\prime}$ in the definition of the coupling is an observable which can be calculated easily through Monte Carlo simulations. Taking the derivative of (4.1) results in

$$
\begin{aligned}
\Gamma^{\prime} & =-\frac{\partial}{\partial \eta} \ln \left\{\int \mathscr{D}[U, \bar{\Psi}, \Psi] \exp \{-S[U, \bar{\Psi}, \Psi]\}\right\} \\
& =\frac{1}{Z} \int \mathscr{D}[U, \bar{\Psi}, \Psi]\left(\frac{\partial S_{\mathrm{G}}}{\partial \eta}+\frac{\partial S_{\mathrm{F}}}{\partial \eta}\right) \exp \{-S[U, \bar{\Psi}, \Psi]\} \\
& =\left\langle\frac{\partial S_{\mathrm{G}}}{\partial \eta}\right\rangle+\left\langle\frac{\partial S_{\mathrm{F}}}{\partial \eta}\right\rangle .
\end{aligned}
$$

Explicit expressions for both expectation values in (4.7) can be found in [11,25]. The calculation of the renormalized coupling $\bar{g}^{2}$ involves expectation values of a local operator and no correlation 
functions. Therefore the numerical evaluation on a computer is straightforward once configurations are available. The relation to the QCD coupling in the Schrödinger functional scheme $\alpha_{\mathrm{SF}}$ is given by

$$
\alpha_{\mathrm{SF}}(\mu)=\frac{\bar{g}^{2}(L)}{4 \pi}, \quad \mu=1 / L .
$$

To complete the definition of the coupling, also the boundary conditions for the quark fields have to be specified. We follow exactly [23]. In particular the angle entering the spatial periodicity of the quarks is chosen as $\theta=\pi / 5$ since this value is advantageous for the numerical simulations $[11,23]$.

\section{The step scaling function}

The concept of the step scaling function which was introduced in [18] has proven to be a very useful recursive technique to scale the coupling to high energies. As we discussed before, in our finite-volume scheme, the energy scale $\mu$ is identified with $L^{-1}$. Hence the renormalization group function $\beta$ (3.2) describes how the coupling changes if the box size is varied infinitesimally. The step scaling function $\sigma(s, u)$, in comparison, gives then a description how the coupling behaves when the box size $L$ is scaled by a factor $s$

$$
\bar{g}^{2}(s L)=\sigma\left(s, \bar{g}^{2}(L)\right) .
$$

The function $\sigma(s, u)$ can be regarded as an integrated form of the renormalization group $\beta$ function. With the help of the step scaling function (5.1) the coupling can be traced to scales $2^{-k} L_{\max }$ (small box sizes, high energies) starting with an initial value $L=L_{\max }$. The value $s=2$ is commonly used $[11,18,26]$ in the application of $\sigma(s, u)$ and we will also make this choice (from now on $s=2$ and we will drop the argument $s$ ). The relation between the renormalization group $\beta$-function and the step scaling function $\sigma\left(\bar{g}^{2}(L)\right)$ is given by

$$
-2 \ln (2)=\int_{u}^{\sigma(u)} \frac{d x}{\sqrt{x} \beta(\sqrt{x})} .
$$

For small values of the coupling $u$, the step scaling function has the following perturbative expansion

$$
\sigma(u)=u+s_{0} u^{2}+s_{1} u^{3}+s_{2} u^{4}+\cdots
$$

where the coefficients are given by

$$
\begin{aligned}
& s_{0}=2 b_{0} \ln (2), \\
& s_{1}=\left[2 b_{0} \ln (2)\right]^{2}+2 b_{1} \ln (2), \\
& s_{2}=\left[2 b_{0} \ln (2)\right]^{3}+10 b_{0} b_{1}[\ln (2)]^{2}+2 b_{2} \ln (2) .
\end{aligned}
$$

Both Eq. (5.2) with the truncated $\beta$ function as well as the expansion (5.3) can be used as the perturbative approximation of the step scaling function. They differ from each other by higher order terms. We take the first option when we compare our Monte Carlo results with perturbation theory.

To study $\sigma(u)$ numerically one starts with choosing several lattice sizes $L / a$ and tuning the bare coupling $g_{0}^{2}$ and the hopping parameter $\kappa$ in such a way that the value of the renormalized 
coupling $\bar{g}^{2}(L)$ reaches some chosen value $\bar{g}^{2}(L)=u$ and the quark mass vanishes since we are in a massless scheme. The next step is to take $L / a \rightarrow 2 L / a$ and simulate at the same bare parameters. The obtained coupling $\bar{g}^{2}(2 L)$ from the latter simulations is a lattice approximation $\Sigma(u, a / L)$ of the continuum step scaling function $\sigma(u)$. An extrapolation to the continuum of the data points at the same coupling $u$ but growing $L / a$ then leads to one value of the continuum function $\sigma(u)$. The procedure is repeated until a suitable range of $u$ is covered. An appropriate functional description of the continuum step scaling function can be given in the end in the form of a suitable fit-function that interpolates the data.

The lattice approximation $\Sigma(u, a / L)$ of the step scaling function contains remnant lattice effects of order $a$. The reason is that beside our non-perturbative value for $c_{\mathrm{sw}}$ there are boundary improvement coefficients for which only perturbative estimates are available. We use those to the known order $[8,23,24]$ which ensures that $\mathcal{O}(a)$ cutoff effects in the step scaling function appear only starting at three-loop order.

The details of cutoff effects also depend on how the condition of a massless scheme is exactly implemented at a finite lattice spacing. As in the $N_{\mathrm{f}}=2$ computation, we define the massless point on the smaller of the pair of lattices entering the step scaling function. More precisely, the (unrenormalized) PCAC quark mass,

$$
m\left(x_{0}\right)=\frac{\frac{1}{2}\left(\partial_{0}^{*}+\partial_{0}\right) f_{\mathrm{A}}\left(x_{0}\right)+c_{\mathrm{A}} a \partial_{0}^{*} \partial_{0} f_{\mathrm{P}}\left(x_{0}\right)}{2 f_{\mathrm{P}}\left(x_{0}\right)}
$$

at $x_{0}=\frac{T}{2}$,

$$
m_{1}=m\left(\frac{T}{2}\right)
$$

is set to zero. The definition of the correlation functions $f_{\mathrm{A}}, f_{\mathrm{P}}$ is found e.g. in [17,27]. The second reference also contains a discussion of the influence of choosing the massless point in a way which differs from (5.8) at finite lattice spacing. For the improvement coefficient $c_{\mathrm{A}}$ in (5.7) we used the 1-loop result [28].

In addition to the various improvement terms in the action and $c_{\mathrm{A}}$ in (5.7) we exploit our knowledge of $\delta_{1}$ and $\delta_{2}$ from the perturbative calculation of

$$
\begin{aligned}
& \delta(u, a / L)=\frac{\Sigma(u, a / L)-\sigma(u)}{\sigma(u)}=\delta_{1}(a / L) u+\delta_{2}(a / L) u^{2}+\cdots, \\
& \delta_{1}(a / L)=\delta_{10}(a / L)+\delta_{11}(a / L) N_{\mathrm{f}}, \\
& \delta_{2}(a / L)=\delta_{20}(a / L)+\delta_{21}(a / L) N_{\mathrm{f}}+\delta_{22}(a / L) N_{\mathrm{f}}^{2}
\end{aligned}
$$

with the coefficients taken from [26]. For $N_{\mathrm{f}}=4$ we have

\begin{tabular}{lll}
\hline$L / a$ & $\delta_{1}$ & $\delta_{2}$ \\
\hline 4 & -0.0102 & 0.0073 \\
6 & -0.0045 & 0.0013 \\
8 & -0.0024 & 0.00013 \\
\hline
\end{tabular}

Using these data we form the lattice step scaling function [29]

$$
\Sigma^{(2)}(u, a / L)=\frac{\Sigma(u, a / L)}{1+\delta_{1}(a / L) u+\delta_{2}(a / L) u^{2}}
$$


which we expect to have smaller overall cutoff effects. They still start at order $a \times u^{4}$ but terms of order $a^{m} \times u^{n}$ are removed for all $m$ and for $n \leqslant 3$ (in fact non-perturbatively in $a$ ). As mentioned previously, the order $a \times u^{4}$ terms are due to the only perturbatively known boundary improvement terms. Their influence was explicitly checked for $N_{\mathrm{f}}=2$ and found to be minor [11], such that also here we assume that the step scaling function converges effectively at a rate

$$
\Sigma^{(2)}(u, a / L)=\sigma(u)+\mathcal{O}\left(a^{2}\right) .
$$

Once the non-perturbatively determined continuum step scaling function $\sigma(u)$ is known, the running of the coupling can be computed easily. For this purpose, one solves the recurrence

$$
u_{i}=\sigma\left(u_{i+1}\right), \quad i=0, \ldots, n, \quad u_{0}=u_{\max }=\bar{g}^{2}\left(L_{\max }\right),
$$

where $u_{\max }$ is chosen such that the corresponding scale $L_{\max }$ is in the hadronic regime of QCD. Proceeding in this way the coupling can be obtained over a wide range of energies. At a sufficiently large energy $\mu=2^{k} / L_{\max }(k \lesssim n)$, perturbation theory can be applied for determining the quantity $\Lambda L_{\max }$ using (3.7) with the $\beta$ function truncated at 2-loop and 3-loop respectively.

\section{Numerical computation and results}

\subsection{Simulation parameters and raw data}

The choice of the improvement coefficients $c_{\mathrm{t}}, \tilde{c}_{\mathrm{t}}$ and $c_{\mathrm{sw}}$ were as discussed in our preceding paper [17]. The range of $\beta$ for our simulations was limited by the validity range of $c_{\mathrm{sw}}$ with four flavors $(\beta \leqslant 5.0)[17]$. Since our code allows only an even number of lattice points in each direction and lattices beyond $L / a=16$ are too time-consuming for our present resources, we chose lattices $(T=L) L / a=4,6,8$. We picked a sufficient number of values of $\beta \in[5.0,9.5]$ to adequately map out a range $\bar{g}^{2}(L)=0.9 \ldots 2.7, \bar{g}^{2}(2 L)=1.0 \ldots 3.5$. With some tuning of the hopping parameter $\kappa$ the quark mass was kept small enough $\left(\left|m_{1} L\right| \leqslant 0.005\right)$ such that masseffects in the step scaling function are negligible.

We performed our simulations on 4-5 crates of the apeNEXT machine at DESY Zeuthen over a period of about a year. The raw data are listed in Appendix A.

\subsection{Analysis of data}

The computation of the step scaling function on the lattice as described in Section 5 requires $\bar{g}^{2}(L)$ to be fixed to certain values $u$ while the resolution $a / L$ is changed. Previously this was realized by tuning $\beta$ for each pair $u, L / a[8,9,11]$. Instead we here follow the more convenient proposal of [13] to pick a sufficient range and number of bare couplings for each considered $L / a$ and interpolate the running coupling $\bar{g}^{2}(\beta, L / a)$ with a smooth function of $\beta$. Afterwards the function allows access to any value of $\bar{g}^{2}$ in the covered range. As an interpolation we took

$$
\bar{g}^{2}(\beta, L / a)=\frac{6}{\beta}\left[\sum_{m=0}^{n} c_{m, L / a}\left(\frac{6}{\beta}\right)^{m}\right]^{-1}
$$

motivated by perturbation theory. Note, however, that we do not fix the known perturbative expansion coefficients; we do not even require $c_{0, L / a}=1$. Somewhat different forms and values $n$ were checked and we verified that our results do not depend on the details of these interpolations. An example is shown in Fig. 1 for a pair of lattice sizes, namely $L / a=8$ and $L / a=16$. The 


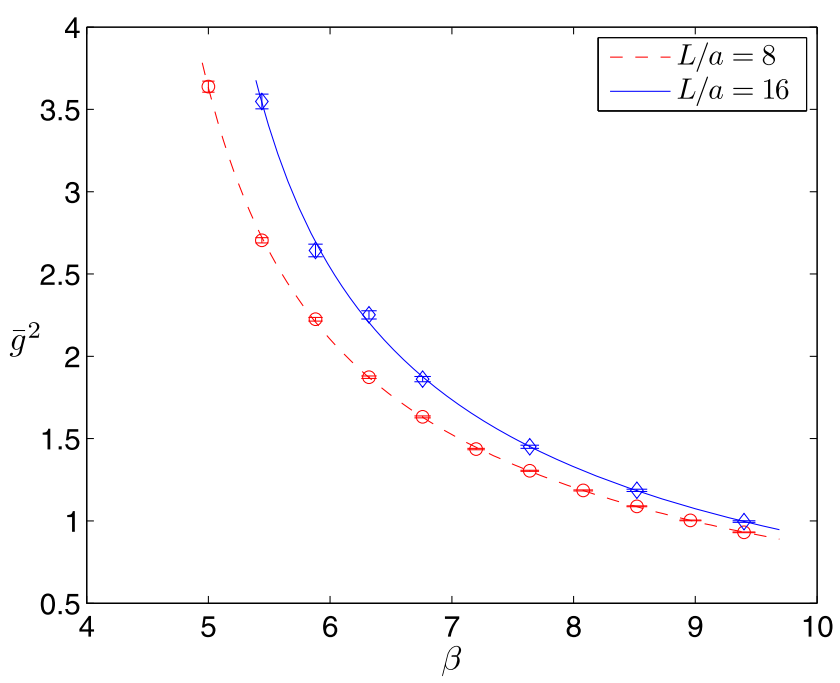

Fig. 1. Data points for $L / a=8$ and $L / a=16$ and their interpolations. The parameter $n$ in (6.1) is set to three. The hopping parameter $\kappa$ was tuned only on the small lattices $L / a=4,6,8$ such that the PCAC mass (5.8) vanished.

coefficients $c_{m, L / a}$ were determined by a standard $\chi^{2}$ fit. From the interpolated couplings we computed $\Sigma(u, a / L)$ for a number of values $u$ starting from an initial coupling $u_{\text {initial }}=0.9$ and following roughly a sequence given by the recursion (5.14).

\subsubsection{Error propagation}

The uncertainties of the initial MC data which can be found in Appendix A are statistically uncorrelated. For the purpose of propagating their errors, let us collect them in a vector $x$ with components $x_{i}$. The above fit function $\bar{g}^{2}(\beta, L / a)$ may then be regarded a function $f(x)$ of the initial data. ${ }^{1}$ The error $\delta f$ of $f$ is simply estimated by

$$
(\delta f)^{2}=\sum_{i}\left(\frac{\partial f}{\partial x_{i}}\right)^{2}\left(\delta x_{i}\right)^{2} .
$$

Since $f$ is a relatively complicated function, it is convenient to avoid computing the derivatives $\partial f / \partial x_{i}$ analytically. Instead we just compute them by a symmetric finite difference, with the step in $x_{i}$ given by $\delta x_{i}$ itself. This convenient method is applied for estimating the errors of all quantities derived from our data in the following. If desired also the correlation matrix of the errors of different observables can be obtained this way.

\subsection{Results}

Our result for both step scaling functions $\Sigma(u, a / L)$ and the perturbatively corrected $\Sigma^{(2)}(u, a / L)$ are listed in Table 1. As one can see in the visualization of our data, Fig. 2, the cutoff effects seem to be very small except for $L / a=4$. As a precaution against higher order

\footnotetext{
${ }^{1}$ We neglect that in the way we determine the interpolation of $\bar{g}^{2}$ there is also a dependence on the uncertainties $\delta x_{i}$. In the fit we could also replace the errors $\delta x_{i}$ by a smooth predefined function of $\beta$. The results would not change significantly.
} 
Table 1

Results for $\Sigma(u, a / L)$ and $\Sigma^{(2)}(u, a / L)$ for different lattices and couplings $u$.

\begin{tabular}{llllllll}
\hline$u$ & $L / a$ & $\Sigma(u, a / L)$ & $\Sigma^{(2)}(u, a / L)$ & $u$ & $L / a$ & $\Sigma(u, a / L)$ & $\Sigma^{(2)}(u, a / L)$ \\
\hline 0.93 & 4 & $0.995(2)$ & $0.999(2)$ & 1.4435 & 4 & $1.608(3)$ & $1.608(3)$ \\
& 6 & $1.000(3)$ & $1.004(3)$ & & 6 & $1.627(6)$ & $1.633(6)$ \\
1 & 8 & $0.995(5)$ & $0.997(5)$ & & 8 & $1.632(8)$ & $1.637(8)$ \\
& 4 & $1.076(2)$ & $1.079(2)$ & 1.6285 & 4 & $1.844(5)$ & $1.839(5)$ \\
& 6 & $1.083(3)$ & $1.086(3)$ & & 6 & $1.868(8)$ & $1.875(8)$ \\
1.0813 & 8 & $1.079(4)$ & $1.081(4)$ & & 8 & $1.874(11)$ & $1.880(11)$ \\
& 4 & $1.171(2)$ & $1.174(2)$ & 1.8700 & 4 & $2.169(8)$ & $2.155(8)$ \\
1.1787 & 6 & $1.179(4)$ & $1.183(4)$ & & 6 & $2.199(13)$ & $2.208(13)$ \\
& 8 & $1.178(5)$ & $1.181(5)$ & & 8 & $2.203(17)$ & $2.212(17)$ \\
1.2972 & 6 & $1.286(2)$ & $1.287(2)$ & 2.2003 & 4 & $2.650(11)$ & $2.617(11)$ \\
& 8 & $1.297(5)$ & $1.301(5)$ & & 6 & $2.688(17)$ & $2.698(17)$ \\
& 6 & $1.298(6)$ & $1.301(6)$ & & 8 & $2.684(24)$ & $2.697(24)$ \\
& 8 & $1.428(3)$ & $1.430(3)$ & 2.6870 & 4 & $3.462(22)$ & $3.378(21)$ \\
\end{tabular}

Table 2

Results of different fit procedures (as described in the text) for the continuum extrapolation of the step scaling function.

\begin{tabular}{llll}
\hline$u$ & $\sigma(u)$ & & \\
\cline { 2 - 4 } & constant fit & global fit & $L / a=8$ data \\
\hline 0.9300 & $1.002(3)$ & $1.002(3)$ & $0.997(5)$ \\
1.0000 & $1.084(3)$ & $1.084(3)$ & $1.081(4)$ \\
1.0813 & $1.182(3)$ & $1.182(4)$ & $1.181(5)$ \\
1.1787 & $1.301(4)$ & $1.301(5)$ & $1.301(6)$ \\
1.2972 & $1.448(5)$ & $1.448(7)$ & $1.450(7)$ \\
1.4435 & $1.634(5)$ & $1.634(10)$ & $1.637(8)$ \\
1.6285 & $1.877(7)$ & $1.877(16)$ & $1.880(11)$ \\
1.8700 & $2.209(10)$ & $2.207(27)$ & $2.212(17)$ \\
2.2003 & $2.698(14)$ & $2.694(49)$ & $2.697(24)$ \\
2.6870 & $3.507(30)$ & $3.50(10)$ & $3.496(44)$ \\
\hline
\end{tabular}

cutoff effects, we thus excluded the data set of our coarsest lattice from our analysis leading to the continuum step scaling function. We carried out three different analysis.

- Constant fit: A fit of $\Sigma^{(2)}(u, a / L)$ for $L / a=6,8$ to a constant, for each $u$.

- Global fit: A fit

$$
\Sigma^{(2)}(u, a / L)=\sigma(u)+\rho u^{4}(a / L)^{2},
$$

with a separate, independent parameter $\sigma(u)$ for each value $u$ but a common parameter $\rho$ modelling the cutoff-effects.

- $L / a=8$ data: Using directly $\sigma(u)=\Sigma^{(2)}(u, 1 / 8)$.

The three different ansätze yield results which are in complete agreement with each other as seen in Table 2. The value of $\rho$ in (6.3) comes out to be $\rho=0.007(85)$ which is a good indication that cutoff effects are negligible in the data for $L / a=6,8$. 


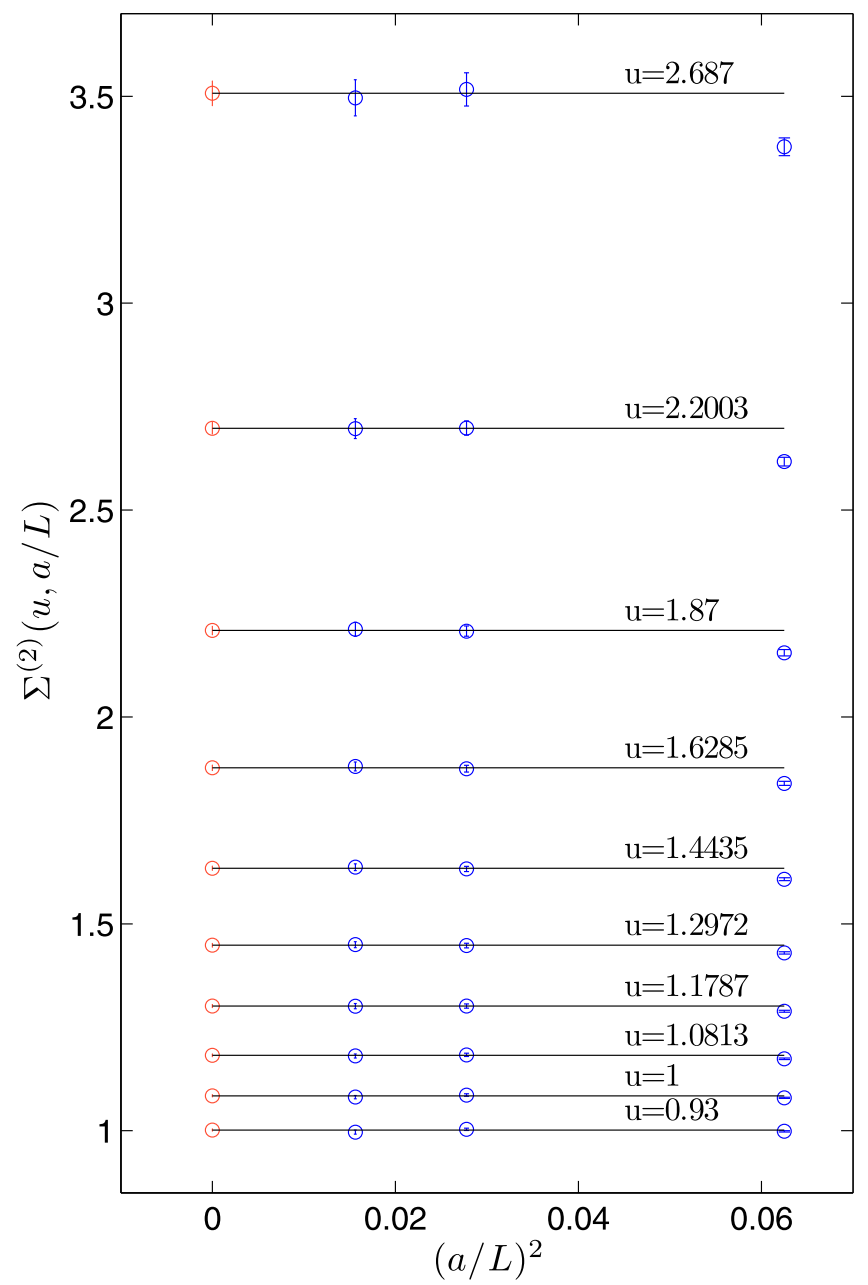

Fig. 2. Continuum extrapolation of the step scaling function (constant fit).

In Fig. 2 we depict the continuum extrapolation with the constant fit. However, as our final results we take just the $L / a=8$ data. This is more conservative and we are confident that the statistical errors dominate over residual cutoff effects. In particular these data agree with the $L / a=6$ data and also using $\Sigma(u, a / L)$ instead of $\Sigma^{(2)}(u, a / L)$ has a negligible effect.

Using a polynomial of degree five in $u$, we performed a constrained interpolation of the data in the fourth column in Table 2 . The coefficients up to $u^{3}$ were fixed by perturbation theory. Our fit

$$
\sigma(u)=u+s_{0} u^{2}+s_{1} u^{3}+0.0036 u^{4}-0.0005 u^{5}, \quad 0 \leqslant u \leqslant 2.7 .
$$

is shown in Fig. 3 (thick line). The perturbative step scaling functions are close to the one sigma range of the non-perturbative data points over the whole interval of the coupling $u$. We note also a peculiarity. Perturbation theory at 3-loop lies below the 2-loop truncation of the $\beta$-function and further away from the non-perturbative result. However, the 3-loop coefficient $b_{2}$ (Eq. (3.6)) in the Schrödinger functional scheme changes its sign between $N_{\mathrm{f}}=2$ and $N_{\mathrm{f}}=3$ and it is rather 


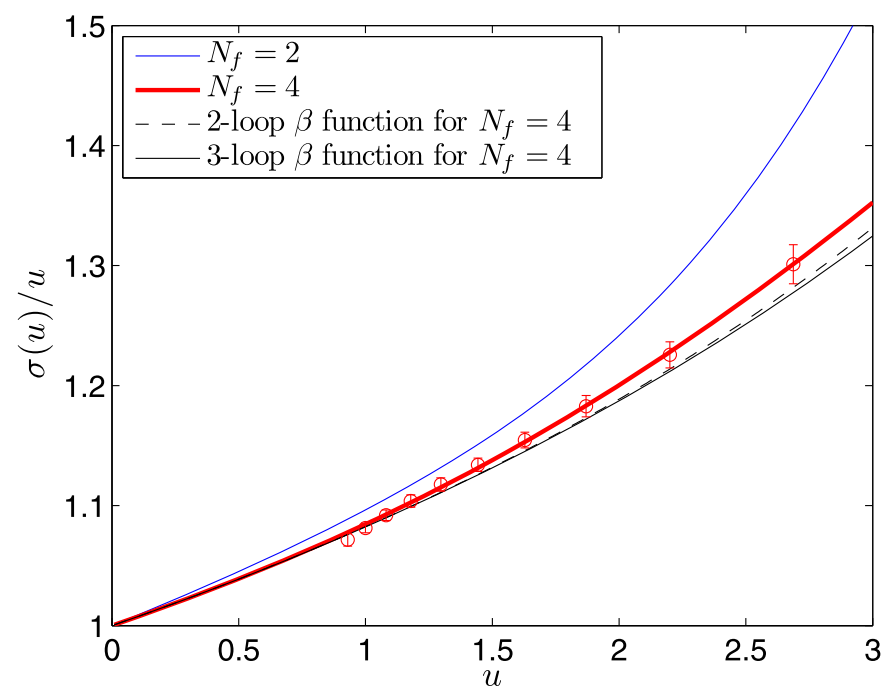

Fig. 3. The step scaling function for $N_{\mathrm{f}}=2,4$ and the perturbative results. The thickest line is the fit of our data points. The upper solid line is the $N_{\mathrm{f}}=2$ result and the lower lines show the perturbative results.

Table 3

Results for $\ln \left(\Lambda L_{\max }\right)$ with different fit strategies.

\begin{tabular}{|c|c|c|c|c|c|c|}
\hline \multirow[t]{2}{*}{$i$} & \multicolumn{2}{|l|}{ constant fit } & \multicolumn{2}{|l|}{ global fit } & \multicolumn{2}{|l|}{$L / a=8$ data } \\
\hline & $u_{i}$ & $\ln \left(\Lambda L_{\max }\right)$ & $u_{i}$ & $\ln \left(\Lambda L_{\max }\right)$ & $u_{i}$ & $\ln \left(\Lambda L_{\max }\right)$ \\
\hline 0 & 3.45 & -2.028 & 3.45 & -2.028 & 3.45 & -2.028 \\
\hline 1 & $2.660(14)$ & $-2.074(17)$ & $2.666(46)$ & $-2.066(56)$ & $2.660(21)$ & $-2.073(26)$ \\
\hline 2 & $2.173(13)$ & $-2.117(24)$ & $2.179(45)$ & $-2.105(83)$ & $2.173(20)$ & $-2.116(37)$ \\
\hline 3 & $1.842(11)$ & $-2.155(28)$ & $1.847(37)$ & $-2.141(97)$ & $1.842(17)$ & $-2.153(44)$ \\
\hline 4 & $1.6013(90)$ & $-2.188(32)$ & $1.606(30)$ & $-2.17(10)$ & $1.602(14)$ & $-2.185(50)$ \\
\hline 5 & $1.4187(78)$ & $-2.217(35)$ & $1.422(25)$ & $-2.20(11)$ & $1.419(13)$ & $-2.213(56)$ \\
\hline 6 & $1.2748(70)$ & $-2.241(39)$ & $1.278(20)$ & $-2.23(11)$ & $1.275(11)$ & $-2.238(63)$ \\
\hline 7 & $1.1583(63)$ & $-2.263(43)$ & $1.161(17)$ & $-2.25(12)$ & $1.159(10)$ & $-2.259(70)$ \\
\hline 8 & $1.0620(58)$ & $-2.282(47)$ & $1.064(15)$ & $-2.27(12)$ & $1.0626(95)$ & $-2.278(76)$ \\
\hline 9 & $0.9809(53)$ & $-2.299(50)$ & $0.982(13)$ & $-2.29(12)$ & $0.9815(87)$ & $-2.294(83)$ \\
\hline 10 & $0.9117(49)$ & $-2.315(54)$ & $0.913(11)$ & $-2.30(12)$ & $0.9122(81)$ & $-2.309(89)$ \\
\hline
\end{tabular}

small for $N_{\mathrm{f}}=4$. It is hence not unlikely that the 4-loop term would move the perturbative curve closer again.

Using the parameterization (6.4) of the step scaling function, we calculated the combination $\ln \left(\Lambda L_{\max }\right)$ starting from the highest coupling $u_{\max }=\bar{g}^{2}\left(L_{\max }\right)$ which was covered by our nonperturbative step scaling function and solved the recursion step (5.14) numerically to obtain the couplings $u_{i}$ which correspond to the energy scales $\mu=2^{i} / L_{\max }$. With the help of (3.7) and using the truncated 3-loop $\beta$ function, we computed the values for $\ln \left(\Lambda L_{\max }\right)$ recorded in Table 3. From the $L / a=8$ results we quote

$$
\ln \left(\Lambda L_{\max }\right)=-2.294(83) \quad \text { at } u_{\max }=3.45
$$

as our final result. This determination of the $\Lambda$-parameter in units of $L_{\max }$ has a precision of $\approx 8 \%$. It remains to gauge $L_{\max }$ in physical units through a large volume computation. Therefore 


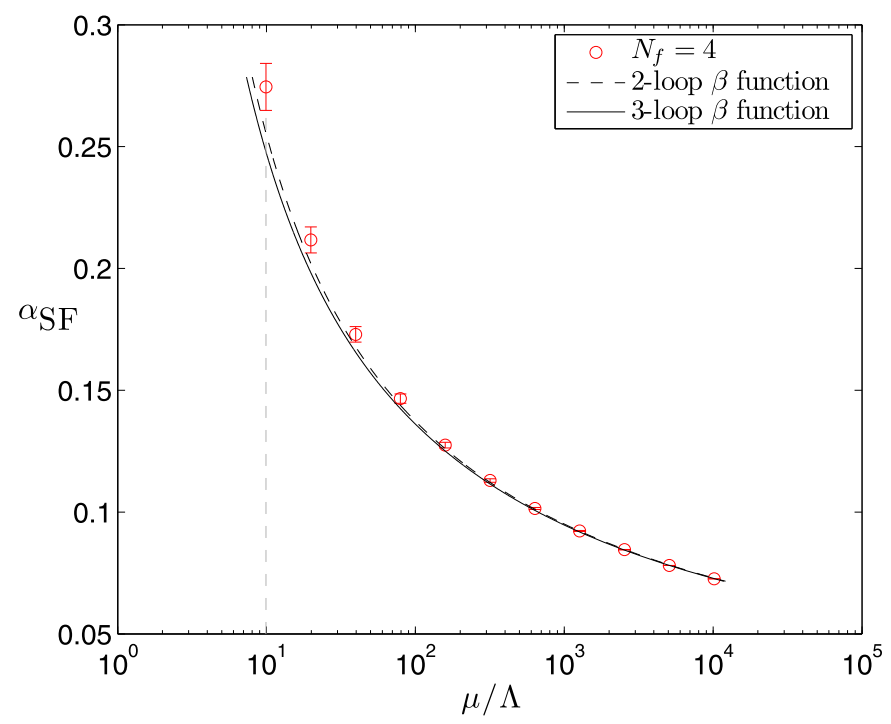

Fig. 4. The running coupling in the Schrödinger functional scheme. The gray vertical dashed line is only for the guidance of the eyes to show what the perturbation theory predicts for $\alpha_{\mathrm{SF}}$ at our lowest energy.

we here show the running of the coupling in the Schrödinger functional scheme in units of $\Lambda$. Fig. 4 displays $\alpha_{\mathrm{SF}}$ computed from the sixth column of Table 3. We observe that upon the iterative application of the step scaling function the difference between the perturbative (using (6.5)) and the non-perturbative coupling is around a 3-sigma effect at the strongest coupling.

\section{Conclusions}

We computed the step scaling function of the QCD coupling in the Schrödinger functional scheme with four massless flavors. We used $\mathcal{O}(a)$ improved Wilson quarks after first determining the Sheikholeslami Wohlert coefficient $c_{\mathrm{sw}}$ [17]. The resulting cutoff effects are very small (Fig. 2, Table 1) allowing for a continuum extrapolation. While the data are compatible with a constant for $L / a \geqslant 6$, we assume this form only for $L / a \geqslant 8$; the smaller lattices thus only enter the analysis by demonstrating that cutoff effects are small. We emphasize that this statement refers to the present level of statistical errors. If in the future statistical errors are further reduced, larger $L / a$ will be necessary at the same time. It will be very interesting to see also the efficiency of computations with different regularizations of the Schrödinger functional as well as the corresponding test of the universality of the continuum limit. Most notably there are chirally rotated boundary conditions for the quarks [30-32] and staggered quarks [33,34] for which results are expected soon.

Already now, we do observe a small but significant deviation from 3-loop perturbation theory at the largest coupling reached in Fig. 4. It is about $10 \%$ (three standard deviations) and the Schrödinger functional coupling has a value of $\alpha_{\mathrm{SF}} \approx 0.28$. For $N_{\mathrm{f}}=2$ a similar effect was visible only for larger coupling [11]. ${ }^{2}$ These findings underline the necessity of going to weak coupling before applying perturbation theory.

\footnotetext{
${ }^{2}$ For $\alpha_{\mathrm{SF}} \approx 0.45$ a similar deviation is visible but there are no non-perturbative data points in between $\alpha_{\mathrm{SF}} \approx 0.28$ and $\alpha_{\mathrm{SF}} \approx 0.45$ to see better where this sets in.
} 
Clearly the present work has brought us a good step closer to the computation of the $\Lambda$ parameter in 4-flavor QCD, which may then be perturbatively connected to e.g. the 5-flavor $\overline{\mathrm{MS}}$ coupling at the Z-pole. However, the technically introduced scale $L_{\max }$ remains to be expressed in physical units through large volume 4-flavor simulations. Apart from the challenge of tuning more parameters, one needs to treat a massive charm quark at small enough lattice spacing. Presently this appears to be a considerable challenge due to a severe slowing down of lattice simulation algorithms at small lattice spacings [35,36].

\section{Acknowledgements}

This work is part of the ALPHA Collaboration research programme. We thank NIC for allocating computer time on the APE computers at DESY, Zeuthen and the staff of the computer center at Zeuthen for their support. This work is supported by the Deutsche Forschungsgemeinschaft (DFG) in the framework of SFB/TR 09 and by the European Community through EU Contract No. MRTN-CT-2006-035482, "FLAVIAnet".

\section{Appendix A. The MC data}

We here list the results of our MC simulations by collecting them in Tables 4-6. Each row refers to a separate simulation of about 50000 trajectories unless otherwise noted. Some of these simulations consist of independent replica (between 1 and 16). Measurements were taken after every trajectory for which we chose trajectory length $\tau=1$.

Table 4

The raw data for $L / a=4$ and $L / a=8$.

\begin{tabular}{|c|c|c|c|c|c|}
\hline \multirow[t]{2}{*}{$\beta$} & \multirow[t]{2}{*}{$\kappa$} & \multicolumn{2}{|l|}{$L / a=4$} & \multicolumn{2}{|l|}{$L / a=8$} \\
\hline & & $\overline{\bar{g}^{2}}$ & $a m_{1}$ & $\overline{\bar{g}^{2}}$ & $a m_{1}$ \\
\hline 5.0 & 0.137975 & $2.913(10)$ & $0.00033(42)$ & $3.932(39)$ & $0.03752(13)$ \\
\hline 5.3 & 0.137110 & $2.4700(76)$ & $0.00040(34)$ & $3.049(22)$ & $0.02934(10)$ \\
\hline 5.6 & 0.136371 & $2.1505(49)$ & $0.00042(29)$ & $2.575(15)$ & $0.024981(80)$ \\
\hline 6.2 & 0.135082 & $1.7300(31)$ & $0.00162(24)$ & $1.9853(91)$ & $0.020192(61)$ \\
\hline 6.8 & 0.134053 & $1.4556(21)$ & $0.00080(20)$ & $1.6256(61)$ & $0.016432(52)$ \\
\hline 7.4 & 0.133188 & $1.2609(13)$ & $0.00032(18)$ & $1.3844(42)$ & $0.013977(47)$ \\
\hline 8.0 & 0.132455 & $1.1119(10)$ & $0.00070(15)$ & $1.2074(32)$ & $0.012435(40)$ \\
\hline 8.6 & 0.131860 & $0.99575(77)$ & $-0.00021(14)$ & $1.0678(25)$ & $0.010382(35)$ \\
\hline 9.2 & 0.131309 & $0.90315(54)$ & $0.00094(11)$ & $0.9662(20)$ & $0.010176(33)$ \\
\hline
\end{tabular}

Table 5

The raw data for $L / a=6$ and $L / a=12$.

\begin{tabular}{|c|c|c|c|c|c|}
\hline \multirow[t]{2}{*}{$\beta$} & \multirow[t]{2}{*}{$\kappa$} & \multicolumn{2}{|l|}{$L / a=6$} & \multicolumn{2}{|l|}{$L / a=12$} \\
\hline & & $\bar{g}^{2}$ & $a m_{1}$ & $\bar{g}^{2}$ & $a m_{1}$ \\
\hline 5.25 & 0.138027 & $2.749(13)$ & $-0.00005(16)$ & $3.635(46)$ & $0.000929(56)$ \\
\hline 5.55 & 0.137173 & $2.3507(92)$ & $0.00110(13)$ & $2.904(29)$ & $0.000704(43)$ \\
\hline 5.85 & 0.136443 & $2.0865(71)$ & $0.00053(11)$ & $2.529(23)$ & $-0.000031(37)$ \\
\hline 6.45 & 0.135190 & $1.6948(46)$ & $-0.000294(94)$ & $1.953(14)$ & $-0.000922(31)$ \\
\hline 7.05 & 0.134123 & $1.4361(32)$ & $0.000488(78)$ & $1.6211(88)$ & $-0.000227(25)$ \\
\hline 7.65 & 0.133261 & $1.2500(24)$ & $0.000437(69)$ & - & - \\
\hline 8.25 & 0.132538 & $1.1025(18)$ & $0.000435(62)$ & $1.2051(50)$ & $-0.000347(20)$ \\
\hline 8.85 & 0.131935 & $0.9908(14)$ & $0.000154(57)$ & - & - \\
\hline 9.45 & 0.131411 & $0.8975(12)$ & $0.000237(51)$ & $0.9628(31)$ & $-0.000547(16)$ \\
\hline
\end{tabular}


Table 6

The raw data for $L / a=8$ and $L / a=16$. The run $L / a=16, \beta=5.44$ has 98000 trajectories.

\begin{tabular}{|c|c|c|c|c|c|}
\hline \multirow[t]{2}{*}{$\beta$} & \multirow[t]{2}{*}{$\kappa$} & \multicolumn{2}{|l|}{$L / a=8$} & \multicolumn{2}{|l|}{$L / a=16$} \\
\hline & & $\overline{\bar{g}^{2}}$ & $a m_{1}$ & $\overline{\bar{g}^{2}}$ & $a m_{1}$ \\
\hline 5.0 & 0.138910 & $3.638(34)$ & $0.00037(14)$ & - & - \\
\hline 5.44 & 0.137507 & $2.705(16)$ & $0.000640(83)$ & $3.548(45)$ & $-0.000872(23)$ \\
\hline 5.88 & 0.136393 & $2.225(11)$ & $0.000306(66)$ & $2.643(38)$ & $-0.001120(25)$ \\
\hline 6.32 & 0.135433 & $1.8728(77)$ & $0.000288(57)$ & $2.252(25)$ & $-0.000875(22)$ \\
\hline 6.76 & 0.134597 & $1.6319(56)$ & $0.000748(58)$ & $1.861(16)$ & $-0.000350(18)$ \\
\hline 7.2 & 0.133903 & $1.4364(42)$ & $0.000041(44)$ & - & - \\
\hline 7.64 & 0.133275 & $1.3046(35)$ & $0.000233(40)$ & $1.4502(94)$ & $-0.000666(15)$ \\
\hline 8.08 & 0.132736 & $1.1852(29)$ & $0.000069(38)$ & - & - \\
\hline 8.52 & 0.132249 & $1.0886(24)$ & $0.000328(36)$ & $1.1860(67)$ & $-0.000552(12)$ \\
\hline 8.96 & 0.131821 & $1.0034(20)$ & $0.000368(33)$ & - & - \\
\hline 9.4 & 0.131442 & $0.9308(17)$ & $0.000284(32)$ & $0.9961(48)$ & $-0.000504(11)$ \\
\hline
\end{tabular}

\section{References}

[1] F. Butler, H. Chen, J. Sexton, A. Vaccarino, D. Weingarten, Hadron masses from the valence approximation to lattice QCD, Nucl. Phys. B 430 (1994) 179, hep-lat/9405003.

[2] S. Aoki, et al., Quenched light hadron spectrum, Phys. Rev. Lett. 84 (2000) 238, hep-lat/9904012.

[3] A. Ali Khan, et al., Light hadron spectroscopy with two flavors of dynamical quarks on the lattice, Phys. Rev. D 65 (2002) 054505, hep-lat/0105015.

[4] S. Aoki, et al., 2+ 1 flavor lattice QCD toward the physical point, Phys. Rev. D 79 (2009) 034503, arXiv:0807.1661.

[5] C. Alexandrou, et al., Light baryon masses with dynamical twisted mass fermions, Phys. Rev. D 78 (2008) 014509, arXiv:0803.3190.

[6] S. Dürr, et al., Ab-initio determination of light hadron masses, Science 322 (2008) 1224, arXiv:0906.3599.

[7] Martin Lüscher, Advanced lattice QCD, Les Houches 1997, Probing the standard model of particle interactions, Pt. 2, 1998, hep-lat/9802029.

[8] Martin Lüscher, Rainer Sommer, Peter Weisz, Ulli Wolff, A precise determination of the running coupling in the SU(3) Yang-Mills theory, Nucl. Phys. B 413 (1994) 481, hep-lat/9309005.

[9] Stefano Capitani, Martin Lüscher, Rainer Sommer, Hartmut Wittig, Non-perturbative quark mass renormalization in quenched lattice QCD, Nucl. Phys. B 544 (1999) 669, hep-lat/9810063.

[10] S. Takeda, et al., A scaling study of the step scaling function in SU(3) gauge theory with improved gauge actions, Phys. Rev. D 70 (2004) 074510, hep-lat/0408010.

[11] Michele Della Morte, et al., Computation of the strong coupling in QCD with two dynamical flavours, Nucl. Phys. B 713 (2005) 378, hep-lat/0411025.

[12] S. Aoki, et al., Precise determination of the strong coupling constant in $\mathrm{N}(\mathrm{f})=2+1$ lattice QCD with the Schrödinger functional scheme, JHEP 0910 (2009) 053, arXiv:0906.3906.

[13] Thomas Appelquist, George T. Fleming, Ethan T. Neil, Lattice study of conformal behavior in SU(3) Yang-Mills theories, Phys. Rev. D 79 (2009) 076010, arXiv:0901.3766.

[14] Q. Mason, et al., Accurate determinations of $\alpha_{s}$ from realistic lattice QCD, Phys. Rev. Lett. 95 (2005) 052002, hep-lat/0503005.

[15] M. Göckeler, et al., A determination of the Lambda parameter from full lattice QCD, Phys. Rev. D 73 (2006) 014513 , hep-ph/0502212.

[16] E. Shintani, et al., Lattice study of the vacuum polarization function and determination of the strong coupling constant, Phys. Rev. D 79 (2009) 074510, arXiv:0807.0556.

[17] Fatih Tekin, Rainer Sommer, Ulli Wolff, Symanzik improvement of lattice QCD with four flavors of Wilson quarks, Phys. Lett. B 683 (2010) 75, arXiv:0911.4043.

[18] Martin Lüscher, Peter Weisz, Ulli Wolff, A Numerical method to compute the running coupling in asymptotically free theories, Nucl. Phys. B 359 (1991) 221.

[19] Particle Data Group, Particle Physics Booklet, http://pdg.lbl.gov, 2008.

[20] Martin Lüscher, Rajamani Narayanan, Peter Weisz, Ulli Wolff, The Schrödinger functional: A renormalizable probe for non-Abelian gauge theories, Nucl. Phys. B 384 (1992) 168, hep-lat/9207009. 
[21] Stefan Sint, On the Schrödinger functional in QCD, Nucl. Phys. B 421 (1994) 135, hep-lat/9312079.

[22] Stefan Sint, One loop renormalization of the QCD Schrödinger functional, Nucl. Phys. B 451 (1995) 416, hep-lat/ 9504005.

[23] Stefan Sint, Rainer Sommer, The running coupling from the QCD Schrödinger functional: A one loop analysis, Nucl. Phys. B 465 (1996) 71, hep-lat/9508012.

[24] Achim Bode, Peter Weisz, Ulli Wolff, Two loop computation of the Schrödinger functional in lattice QCD, Nucl. Phys. B 576 (2000) 517, hep-lat/9911018;

Achim Bode, Peter Weisz, Ulli Wolff, Nucl. Phys. B 600 (2001) 453 (Erratum);

Achim Bode, Peter Weisz, Ulli Wolff, Nucl. Phys. B 608 (2001) 481 (Erratum).

[25] Rainer Sommer, Non-perturbative QCD: Renormalization, $\mathcal{O}(a)$-improvement and matching to heavy quark effective theory, in: Proceedings of the Workshop on Perspectives in Lattice QCD, Nara, Japan (31 October-11 November, 2005), World Scientific, 2008, hep-lat/0611020, 2006.

[26] Bernd Gehrmann, Juri Rolf, Stefan Kurth, Ulli Wolff, Schrödinger functional at negative flavour number, Nucl. Phys. B 612 (2001) 3, hep-lat/0106025.

[27] Achim Bode, et al., First results on the running coupling in QCD with two massless flavors, Phys. Lett. B 515 (2001) 49, hep-lat/0105003.

[28] M. Lüscher, P. Weisz, $\mathcal{O}(a)$ improvement of the axial current in lattice QCD to one-loop order of perturbation theory, Nucl. Phys. B 479 (1996) 429, hep-lat/9606016.

[29] Giulia de Divitiis, et al., Universality and the approach to the continuum limit in lattice gauge theory, Nucl. Phys. B 437 (1995) 447, hep-lat/9411017.

[30] Stefan Sint, The Schrödinger functional with chirally rotated boundary conditions, PoS LAT2005 (2006) 235, heplat/0511034.

[31] Stefan Sint, Lattice QCD with a chiral twist, in: Proceedings of the Workshop on Perspectives in Lattice QCD, Nara, Japan (31 October-11 November, 2005), World Scientific, 2008, hep-lat/0702008, 2007.

[32] Jenifer Gonzalez Lopez, Karl Jansen, Dru B. Renner, Andrea Shindler, Chirally rotated Schrödinger functional: Non-perturbative tuning in the quenched approximation, PoS LAT2009 (2009) 199, arXiv:0910.3760.

[33] Urs M. Heller, The Schrödinger functional running coupling with staggered fermions, Nucl.Phys. B 504 (1997) 435, hep-lat/9705012.

[34] Paula Perez-Rubio, Stefan Sint, The SF running coupling with four flavours of staggered quarks, PoS LAT2007 (2007) 249, arXiv:0710.0583.

[35] Luigi Del Debbio, Haralambos Panagopoulos, Ettore Vicari, Theta dependence of SU(N) gauge theories, JHEP 0208 (2002) 044, hep-th/0204125.

[36] Stefan Schaefer, Rainer Sommer, Francesco Virotta, Investigating the critical slowing down of QCD simulations, PoS LAT2009 (2009), arXiv:0910.1465. 